\title{
Effect of educational intervention based on the Health Belief Model on promoting self-care behaviors of type-2 diabetes patients
}

\author{
Parisa Shabibi ${ }^{1}$, Mohammad Sadegh Abedzadeh Zavareh ${ }^{2}$, Kourosh Sayehmiri $^{3}$, Mostafa Qorbani ${ }^{4,5}$, Omid \\ Safari ${ }^{6}$, Babak Rastegarimehr ${ }^{7}$, Morteza Mansourian ${ }^{8,9}$
}

${ }^{1}$ M.Sc. of Health Education and Promotion, Student Research Committee, Ilam University of Medical Sciences, Ilam, Iran

${ }^{2}$ Ph.D. of Health Education and Promotion, Assistant Professor, Public Health Department, Ilam University of Medical Sciences, Ilam, Iran

${ }^{3}$ Ph.D. of Biostatistics, Associate Professor, Department of Epidemiology, Ilam University of Medical Sciences, Ilam, Iran

${ }^{4}$ Ph.D. of Epidemiology, Assistant Professor, Non-communicable Diseases Research Center, Alborz University of Medical Sciences, Karaj, Iran

${ }^{5}$ Non-communicable Diseases Research Center, Endocrinology and Metabolism Population, Tehran University of Medical Sciences, Tehran, Iran

${ }^{6}$ Pediatrician, Assistant Professor, Departments of Pediatrics, School of Medicine, Alborz University of Medical Sciences, Karaj, Iran

${ }^{7}$ M.Sc., Abadan School of Medical Sciences, Abadan, Iran

${ }^{8} \mathrm{Ph} . \mathrm{D}$. of Health Education and Promotion, Assistant Professor, Health Management and Economics Research Center, Iran University of Medical Sciences, Tehran, Iran

${ }^{9}$ Department of Health Education and Promotion, School of Health, Iran University of Medical Sciences, Tehran, Iran

\section{Type of article: Original}

\begin{abstract}
Background and Objective: Diabetes is a chronic disease in which patients require lifelong self-care behaviors. The present study offset to determine the effect of educational intervention based on the Health Belief Model (HBM) on promoting self-care behaviors of type 2 diabetes patients in Ilam, Iran 2014.

Methods: A quasi-experimental research was conducted based on HBM in which 70 type 2 diabetic patients from Ilam, western Iran in 2014 were selected by multi-stage random sampling. A self-designed questionnaire was used, and pre-test was administered, subsequently, the educational intervention sessions were provided in the form of presentation, questions and answers, group discussion, and practical demonstrations in four sessions over a period of one month. Two months after the intervention, the post-tests were administered. The data were analyzed via SPSS 20 applying independent samples t-test, paired samples t-test, and univariate and multivariate regressions at a significance level of less than 0.05 .

Results: The mean scores of susceptibility, severity, perceived benefits and barriers, self-efficacy, and self-care behaviors were at average and lower levels before the intervention; nonetheless, after the educational intervention, the mean score of each HBM construct and the self-care behaviors significantly increased $(\mathrm{p}<0.001)$.

Conclusion: Health education through HBM promotes the self-care behaviors of patients with type 2 diabetes.

Keywords: Self-Care, Educational Intervention, Diabetes Type 2

\section{Corresponding author:}

Assistant Professor Dr. Morteza Mansourian, Iran University of Medical Sciences, Tehran, Iran.

Tel: +98.2188775409, Fax:+98.2188775410, Email: manssourian55@gmail.com

Received: October 05, 2016, Accepted: September 11, 2017, Published: December 2017

iThenticate screening: September 02, 2017, English editing: October 24, 2017, Quality control: November 02,2017

This article has been reviewed / commented by three experts

(C) 2017 The Authors. This is an open access article under the terms of the Creative Commons Attribution-NonCommercialNoDerivs License, which permits use and distribution in any medium, provided the original work is properly cited, the use is non-commercial and no modifications or adaptations are made.
\end{abstract}




\section{Introduction}

Diabetes is alarmingly increasing across the world (1). Demographic changes and cultural transition of societies and aging in developing countries have turned diabetes into a global epidemic (2); accordingly, the World Health Organization (WHO) has called it a silent epidemic (3). Diabetes is a metabolic disease and a multi-factorial disorder characterized by chronic blood sugar or hyperglycemia, resulting from impaired insulin secretion or action, or both (2). More than $90 \%$ of people with diabetes are individuals with a form of type 2 diabetes (4). The WHO has estimated that the number of patients with diabetes will increase from 135 million in 1995 to 300 million in 2030 (5). The prevalence of type 2 diabetes among Iranian adults (25-64 years old) was estimated as $7.7 \%$ in 2005, and it is predicted that the number of patients with diabetes will reach to more than six million people in 2030 (6). Its complications are undoubtedly on the rise in Iran due to the increasing prevalence of diabetes in (7). It is considered the leading cause of retinopathy, neuropathy, nephropathy, and $60 \%$ of foot amputations (8). In addition, the risk of heart attack and stroke and death from cardiovascular diseases is two to four times greater than other patients in patients with diabetes (9). One of the methods of preventing or delaying the acute and chronic complications is early detection and patients' proper care in order to effectively control and prevent the progression of the disease (2). The successful control of diabetes is dependent upon the patients' self-care because more than $95 \%$ of care associated with diabetes is observed by the patients themselves (10). Self-care is learned, based on the ability of individuals to perform caring practices on their own (11); it can be defined as a strategy to cope with life affairs that promote health and independence, including special activities to alleviate the symptoms of the disease (12). This process is composed of having a healthy nutrition, on-time medication use, blood glucose or urine self-testing, regular exercises, and foot care (13). Studies indicate that adherence to self-care activities improves not only the life quality of patients and their families, but also plays an important role in reducing health care costs because of repeated hospitalizations (3). The improvement of self-care behaviors is the first step to help patients control their disease, making the importance of factors affecting self-care behaviors more obvious (14). One of the main tasks of diabetes care providers is supporting patients to perform their self-care behaviors via giving recommendations for effective self-care diets and providing education to patients (15). Health practitioners encourage patients with diabetes to develop many self-care behaviors (16). Thus, the purpose of the education is for the disease to be managed by the patient and to improve the patients' quality of life (17). To reduce the complications of diabetes, some studies emphasize that healthcare workers should not merely provide knowledge to people, but take into account the perception of the risk as a central concept for understanding healthy behaviors and making changes in behavior. So, patients with diabetes need to properly understand the risk of diabetic complications and the structure of the Health Belief Model (HBM) with respect to constructs appropriate for intervention (18). HBM as a theoretical framework for this research, is one of the most effective models of health education, mainly focused on prevention of diseases and adoption of behaviors to avoid illness and disease chains and it is one of the important precise models which is used to determine the relationship between health beliefs and behaviors. The HBM posits that people will take action to prevent illness if they regard themselves as susceptible to a condition (perceived susceptibility), if they believe it would have potentially serious consequences (perceived severity), if they believe that a particular course of action available to them would reduce the susceptibility or severity or lead to other positive outcomes (perceived benefits), and if they perceive few negative attributes related to the health action (perceived barriers). Additionally, HBM scholars later suggested that self-efficacy-the belief that one can successfully complete the behavior of interest despite considered barriers-be added to the model (19). The HBM Model about type 2 diabetes patients' self-care problems has been considered in the variety of studies $(17,20-21)$. Since diabetes is one of the diseases in which patients have a major role in its control and due to the fact that it is not possible to have the patients under the supervision of healthcare centers at all hours of the day, it is necessary to teach these patients in terms of self-care. In this regard, this study aimed to investigate the effect of educational intervention, based on HBM, on promoting self-care behaviors of type-2 diabetes patients in Ilam, Iran 2014. The conceptual framework of diabetes self-care behavior is illustrated in Figure 1. 


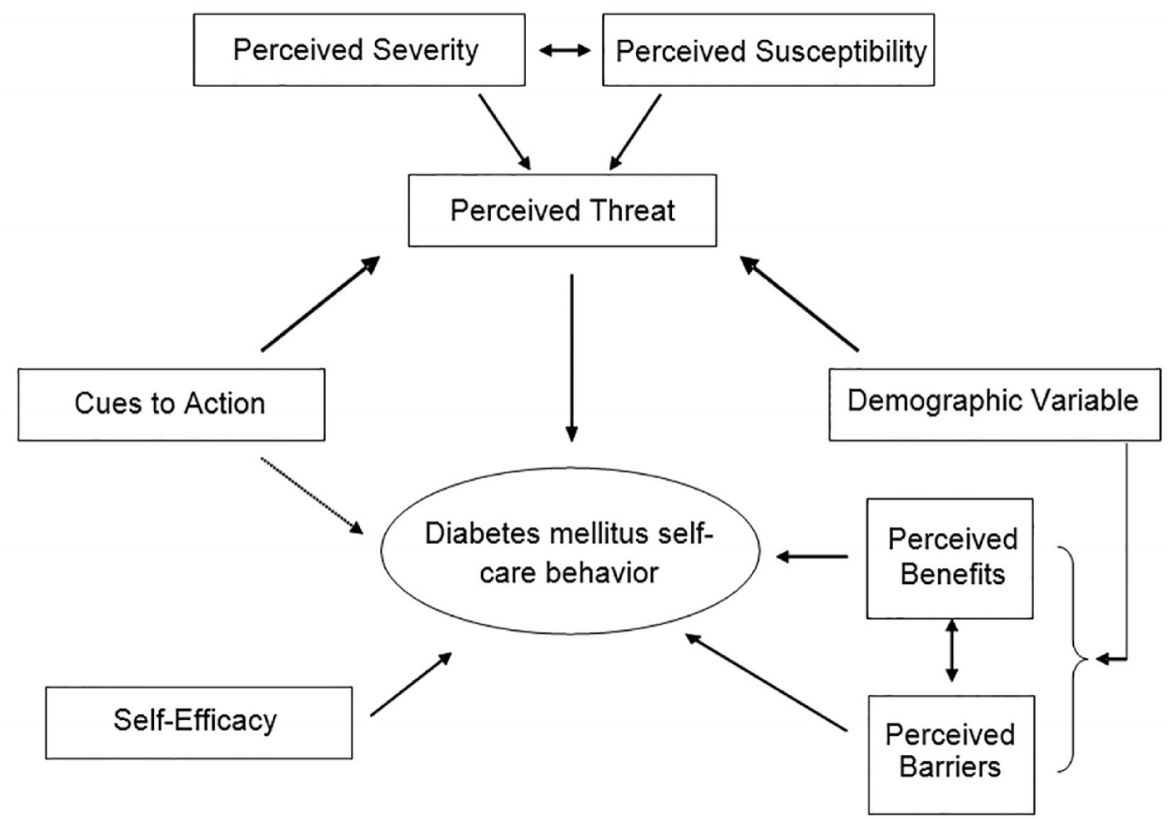

Figure 1. Conceptual framework of diabetes self-care behavior

\section{Material and Methods}

\subsection{Research design and sampling}

A quasi-experimental study was conducted using the intervention method. A sample of 70 patients with type-2 diabetes in rural areas of Ilam, Iran, participated in the study. The sample size according to the formula, to compare the average of the confidence level of $95 \%$ and the test power of $90 \%$ and prediction of $10 \%$ sample reduction, was considered at least 66 people, and finally 70 individuals contributed to the precision of the study. Sampling was conducted by using multi-stage random sampling. Thus, three rural centers were chosen randomly in 2014 between nine rural healthcare centers which are under the Ilam Healthcare Center in 2014. We chose rural health centers because diabetes patient care in the Iranian health system in rural the population is active and in the urban populations is inactive. One health house was randomly selected from each center, and three health houses (Banghelan, Chalesara, and Sartaf) were selected according to the inclusion criteria; Participants' consent was also obtained.

\subsection{Inclusion criteria}

Type-2 diabetic patients with age range between 30 to 60 which are probated by the rural centers in Ilam, stricken to diabetes for more than 6 months, no affection to acute and chronic mental illnesses and being interested in cooperation in the study.

\subsection{Exclusion criteria}

Disinclination to continue participation in the study and the educational program, migration or any other unforeseen reasons such as diseases or hospitalization.

\subsection{Measures}

The tool which is validated for gathering the information is the researcher-made questionnaire with HBM framework. To ensure the validity and reliability of the questionnaire, it was initially examined by a panel of experts including six professors of health education and internal specialists. The validity of the questionnaire was confirmed using the content validity method. The face validity of the questionnaire was also measured employing 10 diabetic patients in rural areas who had not participated in the original investigation. The questionnaire was reviewed in terms of simplicity, fluency, and intelligibility of sentences; and the necessary revisions were made based on their comments. 


\subsection{Validity and reliability}

To test the validity of HBM and self-care constructs, the questionnaire was piloted on 20 patients with diabetes having similar demographic characteristics to the study population. After two weeks, the questionnaire was administered to the same individuals. Test reliability was measured with a test-retest correlation. The reliability values were obtained using the Cronbach's alpha for the constructs of HBM and self-care, and were $89 \%$ and $80 \%$, respectively. The questions were designed in three parts. The first part included 12 questions on patient's demographic information. In the second part, questions were designed and developed to measure each HBM construct (perceived susceptibility six questions, perceived severity five questions, perceived benefits six questions, perceived barriers seven questions, and self-efficacy 10 questions), and a guide to action was used, including four questions based on a 5-item Likert's scale. The Summary of Diabetes Self-Care Activities (SDSCA) which was used in the third part of the standard self-care questionnaire, contained 10 questions. SDSCA is the revised version of Tubert and Glasgow self-care behaviors (22). The reliability of the questionnaire was verified and its validity was translated (23). This questionnaire is employed to assess self-care activities in five main areas: having a diet, doing physical activities, taking medications, monitoring blood sugar, and taking care of the feet. The answers to this questionnaire are designed in a way that the patient may report the amount of his/her activity during the past week, and the answers are scored zero and seven, in which zero stands for no self-care activities during the past week and seven represents performing self-care behaviors in seven days. Rating in the present questionnaire was in a way that the score range for each item of perceived susceptibility and severity, perceived barriers, and self-efficacy varied from 1 to 5 , so $1,2,3,4$, and 5 were awarded to the answers of never, low, medium, high, and very high, respectively. Accordingly, the range for the susceptibility scores is 6 to 30 . The score range for the perceived severity is 5 to 25 . The score range for the perceived barriers is 7 to 35 , and the score range for self-efficacy is 10 to 50. The score range for the perceived benefits is 6 to 35. Regarding perceived benefits, the scores of $5,4,3,2$, and 1 are awarded to strongly agree, slightly agree, neutral, slightly disagree, and strongly disagree, respectively. As much as the score of model constructs goes up, the amount of self-care behavior increases as well. The third part involves 10 questions from the questionnaire of the diabetes self-care behaviors which measures self-care behaviors during the past seven days with a score range of 0 to 70 . Questionnaires were completed in the form of interviews by the researcher. After completing the questionnaire, the data were analyzed.

\subsection{Study intervention}

According to the objectives, available resources, and pre-tests results, the educational need analysis was conducted, and the educational content was developed in three areas of knowledge, attitude, and behavior. The educational methods and instruments and the number of sessions required were determined, and then the intervention based on HBM in the target group, including four 60-minute sessions in a month, was held, i.e. each session was held once a week. The first session contained an introduction on diabetes, symptoms, complications, and the ways to prevent it. The second session was composed of knowledge of self-care aspects (foot care, regular drug use, quitting smoking, and physical activities, particularly walking). The third session included the familiarity of the patients with a healthy diet and proper nutrition. In the fourth session, the patients became familiar with testing blood sugar through practical demonstrations shows by the researcher, and the patients were asked to practice this activity in the session. In this session, the patients reported and discussed their own performance in terms of taking care of the feet, taking medications to control blood sugar, and regularly visiting doctors to have their eyesight, heart, and kidneys examined. At the end of each session, the educational content was given to the patients in the form of educational pamphlets, and before the start of each session, the materials presented were briefly reviewed. The educational methods included presentations, questions and answers, group discussion, and practical demonstrations to help the patients appropriately understand the contents and prevent their misunderstanding. To involve the patients' eyesight and to provide variety, other materials and teaching aids such as instructional videos, posters, and booklets were used. The patients received their special diets by the nutritionist. They also performed the type and the manner of sporting activities useful for diabetic patients in the group and in practice. In the educational sessions, a family member of patients was asked to take part in the sessions, and an educational booklet was provided to increase patients' family support in how to help them control their diabetes. The post-test was administered two months after the training.

\subsection{Ethics of research}

To consider the ethical issues, after providing general information on the objectives and nature of the study, written consents were obtained from all participants. The data were collected confidentially and anonymously and then analyzed. Written informed consent was obtained from the patients for the publication of this report and any accompanying images. The ethics committee code for the study is: IR.Medilam.REC.1395.61 


\subsection{Statistical analysis}

The collected data were analyzed by IBM@ SPSS $\odot$ Statistics version 20 (IBM@ Corp., Armonk, NY, USA), using descriptive statistical methods including absolute frequency distribution and analyzed statistics such as independent samples t-test. In order to compare the mean of scores of HBM constructs before and after, the paired-samples t-test and single variable and multi-variables regression analysis to measure the variables was used. These variables are associated with self-care behaviors. The p-value less than 0.05 was considered as significant.

\section{Results}

In terms of gender, $78.6 \%$ of participants were female, the mean age of participants was $56.59(\mathrm{SD}=9.61)$. The duration mean of the disease among the patients was eight years. Table 1 shows other demographic characteristics of the patients. Table 2 presents the variations of mean in each HBM construct (perceived susceptibility, perceived severity, perceived benefits, perceived barriers and self-efficacy) and self-care behaviors before and after intervention. The mean score of susceptibility, severity, benefits, barriers, self-efficacy and self-care behaviors was average or lower before education, but after the educational intervention, the mean scores of each HBM construct and self-care behaviors increased dramatically. According to the results of Table 2 and paired samples t-test, there is significant difference between the mean scores of HBM constructs and self-care before and after the educational intervention $(p<0.001)$. By increasing the perceived susceptibility, severity, benefits, barriers and self-efficacy, the self-care behaviors increased as well. In this study, the most important guides for the patients were the physicians, healthcare workers and family members. This issue denotes the importance of continuous and regular classes which are held by healthcare personnel for patients. On the other hand, as regard to significant roles of family members in raising the level of self-care as the second guides for patients, the importance of their continuous and regular participation is highlighted. The patients' families who were familiar to educational issues and self-care skills educated them. Using single variable factor analysis, the impact of HBM constructs, i.e. perceived susceptibility, perceived severity, perceived barriers, perceived benefits and self-efficacy before and after the educational intervention, was measured regarding the separation of self-care behaviors of type- 2 diabetic patients. The results showed that HBM constructs were not significantly effective on promoting the self-care behaviors in the type-2 diabetic patients before the educational intervention; but, after the intervention, the significance level of all constructs was less than 5\%. As a result, the HBM constructs were significantly effective on promoting the self-care behaviors in type- 2 diabetic patients after the intervention. The coefficients denote that between the HBM constructs, the self-efficiency with coefficient of affection of (26.6\%) and perceived barriers with coefficient of affection of $(12.1 \%)$ have the highest and least efficacy respectively on promoting self-care behaviors in type-2 diabetic patients.

Table 1. Absolute and relative frequency distribution of the patients in the study according to demographic variables

\begin{tabular}{|l|l|l|l|}
\hline Demographic characteristics & $\mathrm{n}$ & $\%$ \\
\hline Gender & Male & 15 & 21.4 \\
\cline { 2 - 4 } & Female & 55 & 78.6 \\
\hline Education & Uneducated & 43 & 61.4 \\
\cline { 2 - 4 } & Primary School & 18 & 25.7 \\
\cline { 2 - 4 } & Secondary School & 4 & 5.7 \\
\cline { 2 - 4 } & Diploma & 3 & 4.3 \\
\cline { 2 - 4 } & University & 2 & 2.9 \\
\hline Occupation & Governmental & 2 & 2.9 \\
\cline { 2 - 4 } & Private & 5 & 7.1 \\
\cline { 2 - 4 } & Housewife & 55 & 78.6 \\
\cline { 2 - 4 } & Unemployed & 8 & 11.4 \\
\hline Marital status & Married & 53 & 75.7 \\
\cline { 2 - 4 } & Deceased Spouse & 15 & 21.4 \\
\cline { 2 - 4 } & Never Married & 2 & 2.9 \\
\hline Treatment type & Insulin Therapy & 16 & 22.9 \\
\cline { 2 - 4 } & Oral Medication & 54 & 77.1 \\
\hline
\end{tabular}


Table 2. The results of paired sample t-test on the effect of educational intervention on the constructs of the HBM and self-care behaviors in type 2 diabetic patients in the city of Ilam

\begin{tabular}{|c|c|c|c|c|c|}
\hline \multicolumn{2}{|l|}{ Research variables } & Mean & $\mathrm{SD}^{*}$ & $\mathrm{df}^{* *}$ & p-value \\
\hline \multirow[t]{2}{*}{ Perceived susceptibility } & Before intervention & 14.94 & 4.455 & \multirow[t]{2}{*}{69} & \multirow[t]{2}{*}{0.000} \\
\hline & After intervention & 22.46 & 2.785 & & \\
\hline \multirow[t]{2}{*}{ Perceived severity } & Before intervention & 11.64 & 4.170 & \multirow[t]{2}{*}{69} & \multirow[t]{2}{*}{0.014} \\
\hline & After intervention & 17.81 & 2.349 & & \\
\hline \multirow[t]{2}{*}{ Perceived barriers } & Before intervention & 20.56 & 4.738 & \multirow[t]{2}{*}{69} & \multirow[t]{2}{*}{0.025} \\
\hline & After intervention & 14.90 & 2.566 & & \\
\hline \multirow[t]{2}{*}{ Perceived benefits } & Before intervention & 10.33 & 3.642 & \multirow[t]{2}{*}{69} & \multirow[t]{2}{*}{0.032} \\
\hline & After intervention & 28.29 & 1.287 & & \\
\hline \multirow[t]{2}{*}{ Self-efficacy } & Before intervention & 28.36 & 6.541 & \multirow[t]{2}{*}{69} & \multirow[t]{2}{*}{0.012} \\
\hline & After intervention & 36.31 & 5.126 & & \\
\hline \multirow[t]{2}{*}{ Self-care } & Before intervention & 31.44 & 8.89 & \multirow[t]{2}{*}{69} & \multirow[t]{2}{*}{0.001} \\
\hline & After intervention & 49.20 & 5.586 & & \\
\hline
\end{tabular}

*Standard deviation, ** Degree of freedom

\section{Discussion}

The results show the influential effect of educational interventions on the patients. Perceived susceptibility significantly differed before and after the intervention. The results of the present study show the effect of educational interventions on the patients. Perceived susceptibility was one of the considered concepts in this study which significantly differed before and after the intervention. According to Beranth, the glycemic control level improved by promoting the patient's perceived susceptibility (24). In Sharifirad's study, it was denoted that the higher the perceived sensitivity the better the foot care (20). Furthermore, in Shamsi's study it is concluded that improvement in perceived susceptibility leads to increased walking rate in the patients (25). In addition, Baghiani Moghadam's study demonstrated the efficiency of designed educational messages on increasing the perceived susceptibility of the diabetic patients (26). Also, Jahanlou et al. (27) showed the efficiency of education in improving the perceived susceptibility. In this study, the same result was obtained. Perceived severity was one of the considered constructs in this study. Perceived severity is a person's belief to serious threat and the sentiment associated with the deterioration of encountering an illness or irreversible effects which involve the evaluation of medical and clinical outcomes (such as death, disability, and pain) and the possibility of social consequences (such as the impact on work conditions, family life, and social communication) (28). High perceived severity is likely to bring about self-care behaviors. This variable before the educational intervention, was at an average level; after the intervention, it significantly increased. In the study of Mark Daniel, titled "perceived severity and perceived barriers as factors for prediction of self-control glucose in type 2 diabetes" perceived severity was at the moderate level (29). Moreover, the results are consistent with studies of Ghaffari (30), Asadpour (31), Wai et al. (32) and Jahanlou (26), which are based on HBM and represent increasing the mean score of perceived severity in their educational interventions. Tan also reported that being weak, preventive behaviors in diabetic patients were related to low perceived susceptibility and severity (33). Perceived barriers were other considered components of HBM in this study. Perceived barriers include physical, psychological, or financial barriers which impede a person from carrying out proper health behaviors $(34,35)$. The mean score of the perceived barriers declined significantly from after education. The variable of perceived barriers is different from other constructs of the model. Once the educational intervention takes place, the effectiveness of education is revealed when the mean of perceived barriers had been lower than before, because education must provide a background for the patients to understand fewer barriers in the way towards educational purposes. Agha Mollaei declares in his study that perceived barriers significantly decreased after education $(\mathrm{p}<0.001)(36)$. Also, the results which are obtained in this study are consistent with the results of the reduction of the mean scores of the perceived barriers in the studies of Shamsi and Bayati (37), Koch (38), and Asgari (39). Decreasing of the perceived barriers among patients into self-care behaviors helps them to overcome barriers and become ready to do regular and correct self-care behaviors. The mean score of perceived benefits in diabetic patients increased significantly. This increasing could increase the motivation and tendency among patients about self-care behaviors and adherence to them. The findings show consistency with the results of investigations by Karimi et al. (40) and Jedgal et al. (21) who show an increase in the mean scores of perceived benefits in their educational interventions. 
Self-efficacy is one of the most important of HBM constructs. The mean score for self-efficacy significantly increased. Increasing and being meaningful, the mean score of self-care can be known as a consequence of the educational program. For instance, after caring and general protection of the feet, the mean and standard deviation values significantly increased. The study conducted by Borhani et al. (41-42) is in agreement with the results of this study. The results of the present study show that after the intervention, the mean score of the behavior increased and the educated individuals had a better performance towards diabetes. The studies of Ale Yowmi et al. (43), Sarani (44), Taheri Aziz (45) and Jedgal et al. (21) indicated the effect of educational intervention on promoting preventive behaviors and adhering to treatment or the construct of the pattern. The study findings should be considered in the context of its potential limitations, and the major limitation of the present study was lack of control group.

\section{Conclusions}

Teaching diabetic self-care behaviors based on HBM in terms of improving the level of susceptibility, severity, perceived threat, attention to perceived benefits and barriers, and self-efficacy, using a variety of practical and scientific methods, leads to an increase in the level of care among diabetic patients. Consequently, it leads to a significant increase in the self-care score among patients after the intervention. Thus, the effectiveness and efficiency of HBM on adopting self-care behaviors in patients with type-2 diabetes were confirmed via this study.

\section{Acknowledgments:}

This article is adapted from the Master's thesis approved by Ilam University of Medical Sciences. The authors express their gratitude to the vice president of research for financial and executive support. Also, our sincere thanks go to the diabetic patients and all those who participated in this project.

\section{Conflict of Interest:}

There is no conflict of interest to be declared.

\section{Authors' contributions:}

PS, MM and MSA conceived the study and revised the manuscript. MM and MSA participated in study design and wrote drafts of the manuscript. KS and MQ conceived the study and carried out the statistical analysis. OS and BR participated in revision manuscript. All authors read and approved the final manuscript.

\section{References:}

1) Gadsby R. Epidemiology of diabetes. Adv Drug Deliv Rev. 2002; 54(9): 1165-72.

2) Delavari A, Mahdavi Hazaveh A, Norozi Nejad A, Yarahmadi Sh, Taghipour M. The National Diabetes Prevention and Control Programme. 2nd ed. Tehran: Seda Publishing Center; 2003: 24.

3) Abdoli S, Ashktorab K, Ahmadi F, Parvizy M. Barriers and facilitators the empowerment of people with diabetes. Journal of Endocrinology and Metabolism. 2008; 10(5): 455-64.

4) Esteghamati AH, Halabchi F. Exercise therapy in patients with type 2 diabetes. Iran J Diabetes Lipid Disord. 2008; 7(3): 251.

5) Bener A, Zirie M, Janahi IM, Al-Hamaq AO, Musallam M, Wareham NJ. Prevalence of diagnosed and undiagnosed diabetes mellitus and its risk factors in a population-based study of Qatar. Diabetes Res Clin Pract. 2009; 84(1): 99-106. doi: 10.1016/j.diabres.2009.02.003. PMID: 19261345.

6) World Health Organization. Prevalence of diabetes in the Who Eastern Mediterranean Region Diabetes Programme. 2006. Available from: http://www.who.int/diabetes/facts/world_figures/en/index2.html.

7) Funnel MM, Brown TL, Childs BP, Haas LB, Hosey GM, Jensen B, et al. National Standards for Diabetes Selfmanagement Education. Diabetes Care. 2010; 33(1): 97-104.

8) Bell RA, Arcury TA, Snively BM. Diabetes Foot Self-care Practices in a Rural Triethnic Population. Diabetes Education. 2005; 31(1): 75-83. doi: 10.1177/0145721704272859. PMID: 15779248, PMCID: PMC1613259.

9) Mody R, Kalsekar I, Kavookjian J, Lyer S, Rajagopalan R, Pawar V. Economic Impact of Cardiovascular Co-morbidityin Patients with Type 2 Diabetes. J Diabetes Complications. 2007; 21(2): 75-83. doi: 10.1016/j.jdiacomp.2006.02.005. PMID: 17331855.

10) Anderson RM, Funnell M. Facilitating self-care through empowerment. Psychology in Diabetes Care. 2003; 10(4): 69-97.

11) Bidi F, Hassanpour K, Ranjbarzadeh A, Kheradmand A. Effectiveness of Educational Program on Knowledge, Attitude, Self-Care and Life Style in patients with type II diabetes. Journal of Sabzevar University of Medical Sciences. 2013; 19(4): 336-44. 
12) Nadrian H, Morovati Sharifabad MA, Mirzaei A, Bahmanpur K, Moradzadeh R, Shariati AM. Relationship between quality of life, health status and self-care behaviors in patients with rheumatoid arthritis in Yazd. Journal of Ilam University of Medical Sciences. 2011; 19 (2): 15-25.

13) Baqudano IR, Dossantos MA, Martins TA, Zanetti ML. Self-care of Patients with diabetes mellituse cared for at an emergency service in Mexico. Review Latin American Enfermagem. 2010; 18(6): 1195-202.

14) Nadkarni A. Implementation plans and selfmonitoring of blood glucose in diabetics. Dissertation, Michigan University. 2009.

15) Jafarian Amiri SR, Zabihi A, Babaieasl F, Eshkevari N, Bijani A. Self-care Behaviors in Diabetic Patients Referring to Diabetes Clinics in Babol City, Iran. Journal of Babol University of Medical Sciences. 2010; 12(4): 72-8.

16) Hubley J. Patient Education in the Developing World. Patient Education Counseling. 2006; 61(1): 161-4.

17) Karimy M, Abedi AR, Niknami Sh, Aminshokravi F, Tavafian S. Preventing HIV Transmission among the Opiate- Dependent Population in Zarandieh: Evaluation of the HBM-Based Educational Programs. Journal of health Education and Health Promotion. 2013; 1(1): 2-10.

18) Clement S. Diabetes self-management education. Diabetes Care. 1995; 18: 1204-14. doi: 10.2337/diacare.18.8.1204. PMID: 7587866.

19) Rosenstock IM, Strecher VJ, Becker MH. Social learning theory and the Health Belief Model. Health Educ Q. 1988; 15(2): 175-83. PMID: 3378902.

20) Sharifirad G. Hazaveyee S. Mohebi S. The effect ofeducational program based on Health Belief Model on diabetic foot care. International Journal of Diabetes Development Ctries. 2007; 27: 18-23. doi: 10.4103/0973-3930.34753.

21) Jadga Kh, Zareban I, Alizadeh-Siuki H, Izadirad H. The Impact of Educational Intervention Based on Health Belief Model on Promoting Self-Care Behaviors in Patients with Smear Positive Pulmonary TB. Journal of Health Education and Health Promotion Summer. 2014; 2(2): 143-52.

22) Toobert DJ, Hampson SE, Glasgow RE. The summary of diabetes self-care activities measure: results from7 studies and a revised scale. Diabetes Care. 2000; 23(7): 943-50. doi: 10.2337/diacare.23.7.943. PMID: 10895844 .

23) Zareban I, Niknami Sh, Hidarnia A, Rakhshani F, karimy M, Kuhpayehzadeh J, et al. Predictors of selfcare behavior and its effective factors among women's with type 2 diabetes patients in Zahedan via Health Belief model. Journal of Health System Research. 2014: 1797-805.

24) Beranth C. The health belief model applied to glycemic control. The Diabetes Educator. 1999; 21(4): 321 9.

25) Shamsi M, Sharifirad G, Kachoyee A, Hassanzadeh A. The Effect of Educational Program Walking Based on Health Belief Model on Control Sugar in Woman by Type 2 Diabetics. Iranian Journal of Endocrinology \& Metabolism. 2010; 11(5): 490-9.

26) Baghiani Moghadam MH, Taheri GH, Fallah Zadeh H, Parsa M. The effect of instructional designed SMS based on Health Belief Model (HBM) on adoption of self-care behavior of patients with type II diabetes. Modern Care. 2014; 11(1): 10-8.

27) Gahanloo SH, Ghofranipour F, Vafaei M, Kimiagar M, Heidarnia AR, Sobhani AR, et al. Assessment HBM structures along with DBA1C in diabetic patients with optimum and un favorable diabet. Journal of Hormozgan Medical School. 2008; 12(1): 37-42.

28) Cismaru M. Using protection motivation theory to increase the persuasiveness of public service communications. Regina: Saskatchewan Institute of Public Policy; 2006: 5-27.

29) Daniel M, Messer LC. Perceptions of disease severity and barriers to self-care predict glycemic control in aboriginal persons with type 2 diabetes mellitus. Chronic Disease Cancer. 2002; 23: 130-8.

30) Ghafari M. Comparing the efficacy of Health Belief Model and it's integrated model in AIDS education among male high school students in Tehran. Tarbiat Modares University. 2007: 95-187.

31) Asadpour M. Promotion and maintenance of preventive behaviors from HIV, HBV and HCV infections in health care worker with using constructs of Health Belief Model in Precede-Proceed model. Tarbiat Modares University. 2005: 151-232.

32) Wai CT, Wong ML, Ng S. Utility of Health Belief Model in predicting compliance of screening in patients with chronic hepatitis B. Aliment Pharmacology Therapy. 2005; 21(10): 1255-62. doi: 10.1111/j.13652036.2005.02497.x. PMID: 15882247.

33) Tan MY. The relationship of health beliefs and complication prevention behaviors of chinese individuals with Type 2 diabetes mellitus. Diabetes Research Clinic Practice. 2004; 66(1): 71-7. 
34) Becker MH. The health belief model and personal health behavior. Health Education Monographs. 1974; 2: 324-473.

35) Rosenstock IM. The health belief model and preventive health behavior. Health Education Monographs. 1974; 2(4): 354-86.

36) Agha Molaei T, Eftekhar H, Mohammad K. Application of health belief model to behavior change of diabetic patients. Payesh. 2005; 4(4): 263-9.

37) Shamsi M, Bayati A. A survey of the prevalence of self-medication and the factors affecting it in pregnant mothers referring to health centers in Arak city. Jahrom Medical Journal. 2010; 7(3): 34-42.

38) Koch J. The role of exercise in the African- American woman with type 2 diabetes mellitus: application of the Health Belief Model. Journal of American Academic Nurse Practice. 2002; 14(3): 126-9. doi: 10.1111/j.1745-7599.2002.tb00103.x.

39) Asghari T. The effect of educational program safety on based health belief model in workers. Dissertation Esfahan School of Public Health. 2006.

40) Karimi M, Zareban I, Montazrei A, Aminshokravi F. the impact of education based on Health Belif Model in preventive behavior of unwanted pregnancy. IJOGI. 2012; 15(23): 18-27.

41) Dizaji MB, Taghdisi MH, Solhi M, Hoseini SM, Shafieyan Z, Qorbani M, et al. Effects of educational intervention based on PRECEDE model on self-care behaviors and control in patients with type 2 diabetes in 2012. Journal of Diabetes \& Metabolic Disorders. 2014; 13: 72. doi: 10.1186/2251-6581-13-72. PMID: 25075380, PMCID: PMC4114427.

42) Borhani M, Rastgarimehr B, Shafieyan Z, Mansourian M, Hoseini SM, Arzaghi SM, et al. Effects of predisposing, reinforcing and enabling factors on self-care behaviors of the patientswith diabetes mellitus in the Minoodasht city, Iran. J Diabetes Metab Disord. 2015; 14; 14-27.

43) Olayemi SO, Oreagba IA, Akinyede A. Educational intervention and the health seeking attitude and adherence to theraphy by tuberculosis patients from an urban slum in Lagos Niger Postgrad. Niger postgrad Medy. 2009; 16(4): 231-5.

44) Sarani M. The Study for Health Belief Model efficiency in adopting preventive behaviors in the Sistan region tuberculosis patients 2009-2010. Medical Sciences and Health Services Zahedan. 2011; 1: 152-3.

45) Taheriaziz M. Survey of effectiveness of designed health educational package on tuberculosis patient's healthy behaviors at Pasteur institute spring 2004. Tarbiat Modares University. 2004; 1: 231. 